\title{
Entrelacs
}

ENTRELACS Cinéma et audiovisuel

$15 \mid 2018$

Récits de soi

\section{Techniques de soi et technologies numériques}

\section{Bidhan Jacobs}

\section{(2) OpenEdition \\ Journals}

\section{Édition électronique}

URL : http://journals.openedition.org/entrelacs/3005

DOI : 10.4000/entrelacs.3005

ISBN : 2261-5482

ISSN : 2261-5482

Éditeur

Éditions Téraèdre

Référence électronique

Bidhan Jacobs, «Techniques de soi et technologies numériques », Entrelacs [En ligne], 15 | 2018, mis en ligne le 21 octobre 2018, consulté le 19 avril 2019. URL : http://journals.openedition.org/ entrelacs/3005; DOI : 10.4000/entrelacs.3005

Ce document a été généré automatiquement le 19 avril 2019.

Tous droits réservés 


\title{
Techniques de soi et technologies numériques
}

\author{
Bidhan Jacobs
}

1 Le $\mathrm{XXI}^{\mathrm{e}}$ siècle est celui du « numérique intégral » ${ }^{1}$. Pas un domaine de la société, de ses secteurs d'activité et de la vie qui ne soit investi, occupé, converti et façonné par les technologies numériques et réordonné à sa mise en réseau. Cette mutation prodigieuse et unique (jamais dans l'histoire des techniques l'introduction d'une technologie n'aura transformé la société aussi radicalement et avec une telle fulgurance) serait porteuse de nombreux bénéfices pour tous. Les technologies numériques constituent cependant un sous-ensemble d'un vaste système politique, industriel et militaire, vecteur d'un programme économique écrasant. En effet, depuis la fin des années 1970, le numérique est avant tout une arme idéologique entre les mains du néolibéralisme ${ }^{2}$ et, par conséquent, une " technologie politique des individus $»^{3}$. Tout se passe comme si l'exercice du pouvoir à travers les technologies numériques était invisible, inexistant, pouvant faire croire à leur neutralité. Or, le sujet du pouvoir existe pourtant toujours : « Il est précisément partout où il travaille activement à se faire oublier. C'est même cette intense activité d'effacement de lui-même qui le signale immanquablement $»^{4}$. Ces technologies exécutent, avec une grande efficience, un programme politique implacable : stratégies de détection et répression des comportements humains, processus extensif de réduction et d'uniformisation générale des êtres humains à des données, et de conformation irréversible à ce processus. La critique des technologies développée en sciences humaines depuis les travaux de Michel Foucault a contribué à concevoir les outils nécessaires à la compréhension et l'analyse de cette forme de gouvernementalité computationnelle ${ }^{5}$.

2 Le « souci de $\operatorname{soi}^{6}$ » que Foucault voit se développer en Occident depuis l'Antiquité, comme art de se conduire - c'est-à-dire, de se gouverner et de transmettre aux autres - est à la fois « une notion, un précepte, une attitude et un ensemble de techniques : une matrice pratique de l'expérience de soi $^{7} »$. S'il est vrai que le souci de soi doit être considéré comme « le corrélatif de technologies développées à travers notre histoire ${ }^{8}$ ", qu'en est-il de ses transformations induites par les technologies numériques ? La réflexion de 
Foucault considérant début des années 1980 que la disparition apparente de ce thème viendrait de ce que ces techniques de soi « ont été intégrées, dans notre monde, dans des techniques éducationnelles et pédagogiques, médicales et psychologiques, et, [par conséquent] (...) intégrées dans des structures d'autorité et de discipline (...) de sorte que la culture de soi serait imposée aux gens par les autres et qu'elle a perdu son indépendance 9 ", nous autorise à proposer l'hypothèse que ce sont à présent les technologies numériques intégrées à l'ensemble de la société qui ont assimilé et détourné les techniques de soi. Aussi l'une des tâches à laquelle s'attellent certains artistes seraitelle « d'envisager comment il serait possible d'élaborer de nouveaux types, de nouvelles sortes de relations à nous-mêmes ${ }^{10}$ ». Pour ce faire, nous allons tenter d'analyser les techniques et les formes inventées par trois artistes d'aujourd'hui : Leighton Pierce, Sabine Massenet et Jacques Perconte.

Leighton Pierce, photographe, cinéaste, vidéaste, artiste du numérique et enseignant, directeur du CalArts depuis juillet 2013, ne cesse de découvrir de nouvelles formes du flou depuis les années 1980 et travaille dans la droite lignée de Jean Epstein le phénomène de fluidité ${ }^{11}$ : Evaporation (2002) opère une extension et une généralisation de la fluidité rapportée à l'expérience de soi.

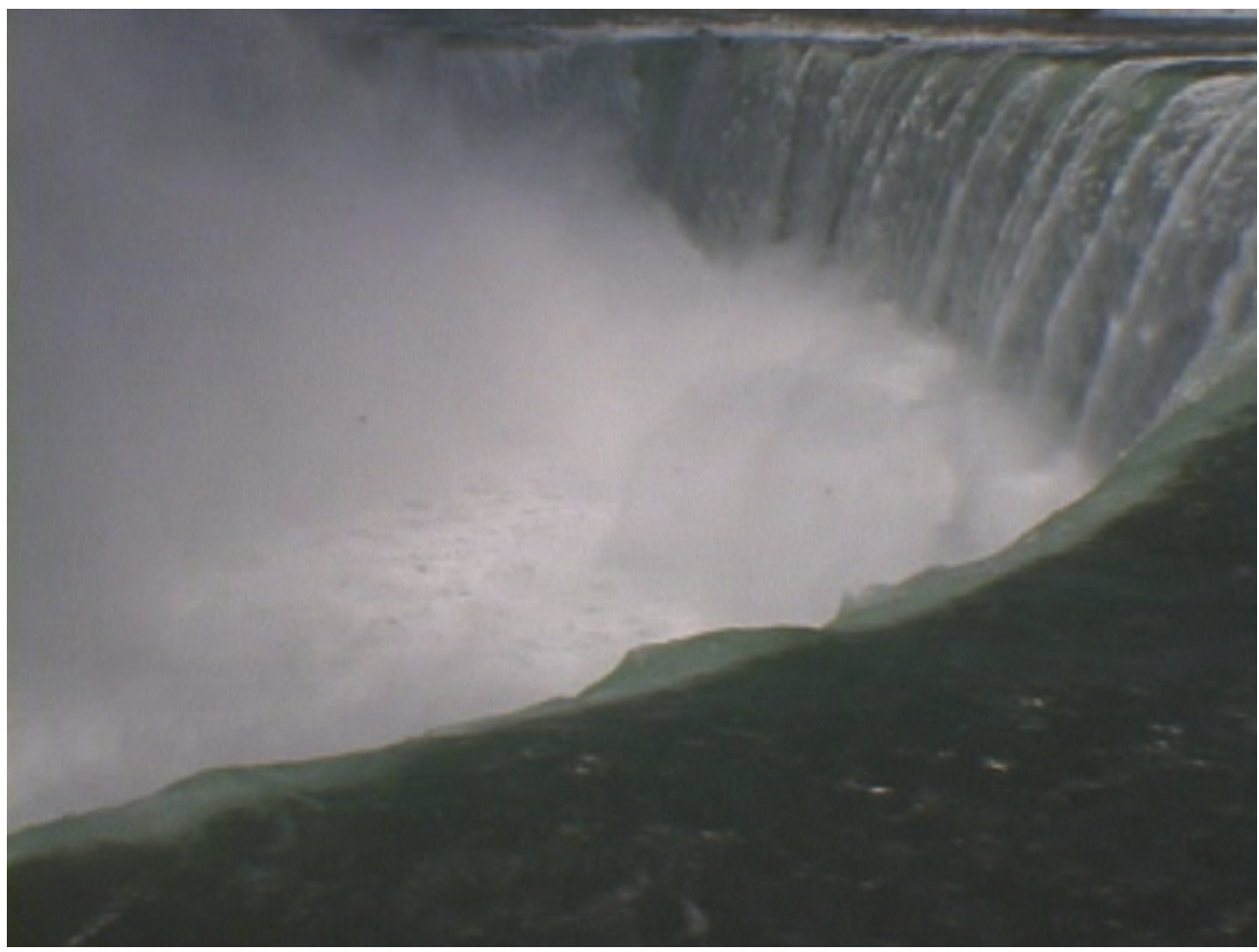

Evaporation, Leighton Pierce (2002)

Le principe physique de l'évaporation de l'eau se décline au travers d'immenses chutes d'eau provoquant de puissantes déflagrations de vapeur froide, de la mer dont les vagues sont hérissées et laissent s'échapper dans des frissons des gouttes d'eau, de l'écume blanche des vagues venant s'échouer sur la plage ou encore jaillissant de la coque d'un bateau fendant les eaux, des vues en plongée verticale, ralentie et en surimpression, des vagues dont les crêtes miroitantes dessinent des ondes aux fréquences élevées et irrégulières, de paysages marins baignés d'une brume dense. 

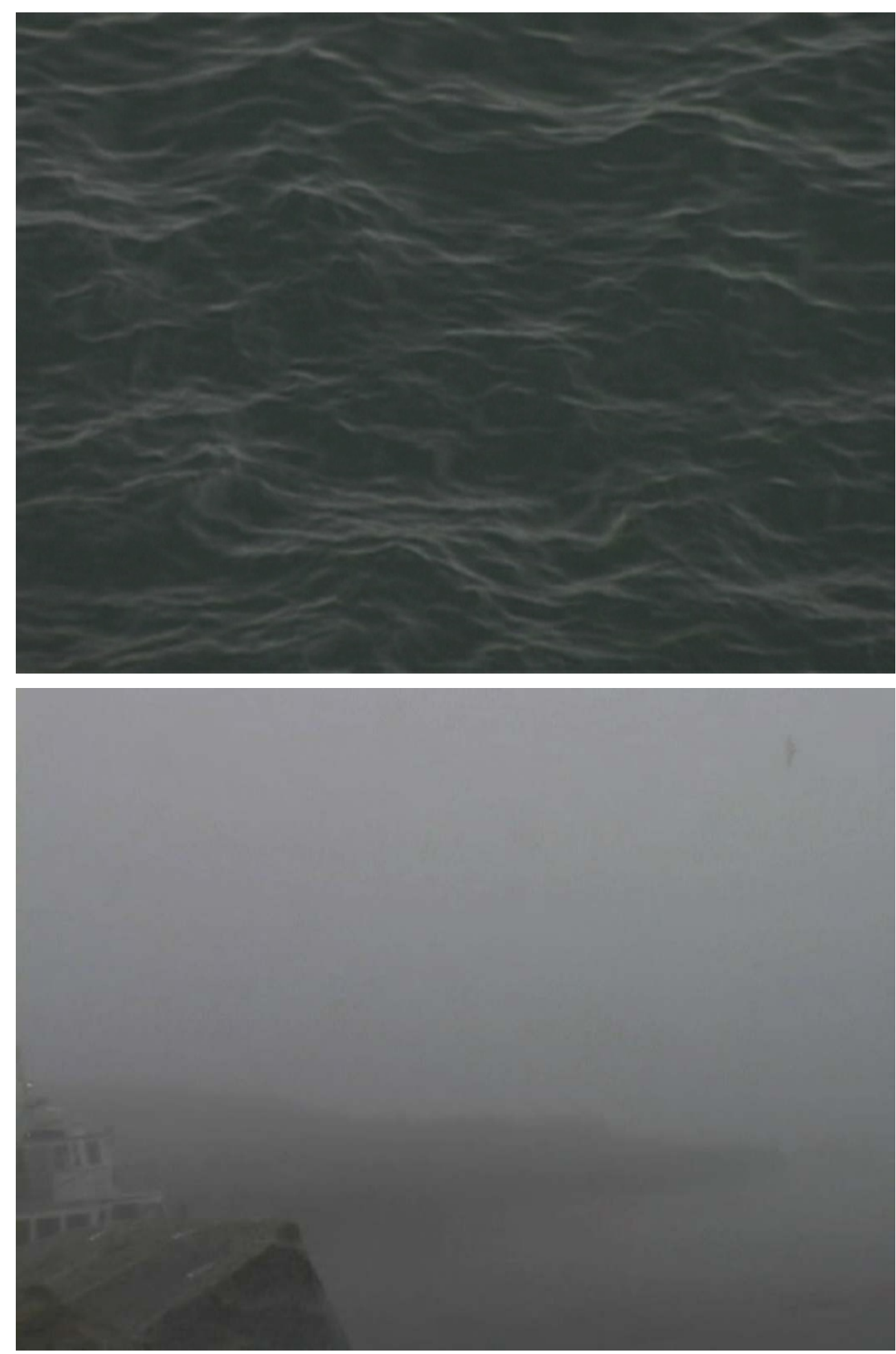

Evaporation, Leighton Pierce (2002)

5 Ce principe physique, qui constitue donc une ressource plastique infinie, finit par affecter toutes les matières, garçons, herbes, fleurs, arbres. En effet, Pierce joint deux techniques : d'une part, l'exploitation de flous avant très élevés ${ }^{12}$; et de l'autre, ce qu'il nomme le smearing, pratique complexe qui combine la très faible vitesse d'obturation (qui produit des flous temporels) à une technique de montage de son invention, sous Final Cut Pro, démultipliant quatre à huit fois le plan tourné afin d'obtenir par le décalage des pistes et 
les transparences une séquence élaborée de traînées de mouvement et de couleurs qui s'enchevêtrent dans des entrelacs si précis et si imperceptibles que l'ensemble se meut à la manière d'un chaos doux et harmonieux ${ }^{13}$. Ainsi toutes les formes deviennent-elles évanescentes, vaporeuses, ondulatoires et se tordent comme sous l'effet de complexes convections de l'air. Pierce rapporte, tout d'abord, l'évaporation à ce qu'elle est réellement : au changement d'état de l'eau, par la chaleur, le froid, la force des flux, d'immenses cycles naturels. Mais il l'indexe à la dynamique des fluides en général, à laquelle même les solides sont soumis puisqu'ils sont polarisés dans le temps ainsi que Epstein l'a déjà démontré dans ses écrits et ses films ${ }^{14}$; enfin, aux changement d'état mentaux de ses enfants adolescents et de son propre rapport à eux : non pas dissolution des rapports, mais leurs continuelles transformations, parce que l'être humain est aussi labile, physiquement et psychiquement, que tous les éléments de la nature.

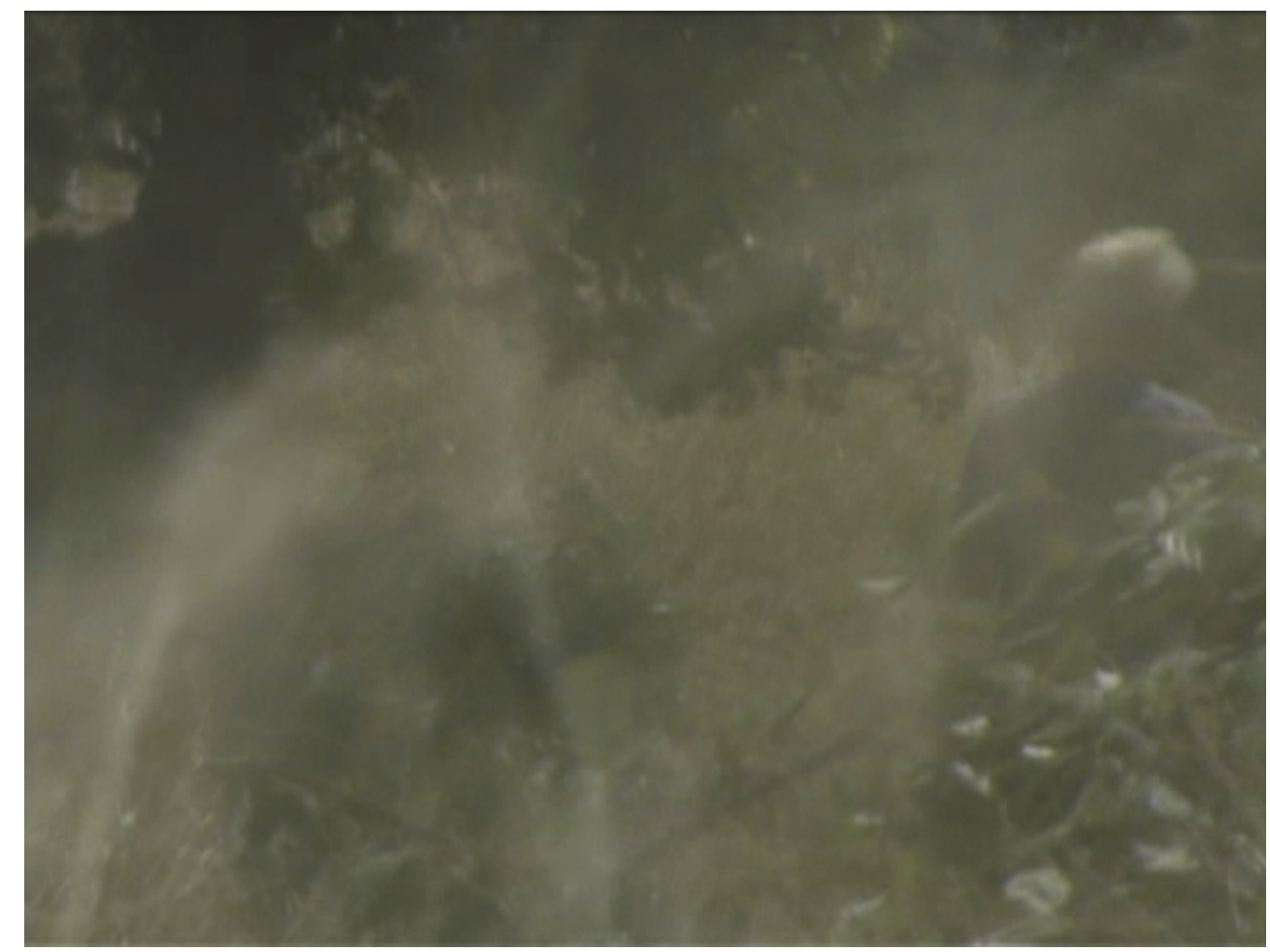




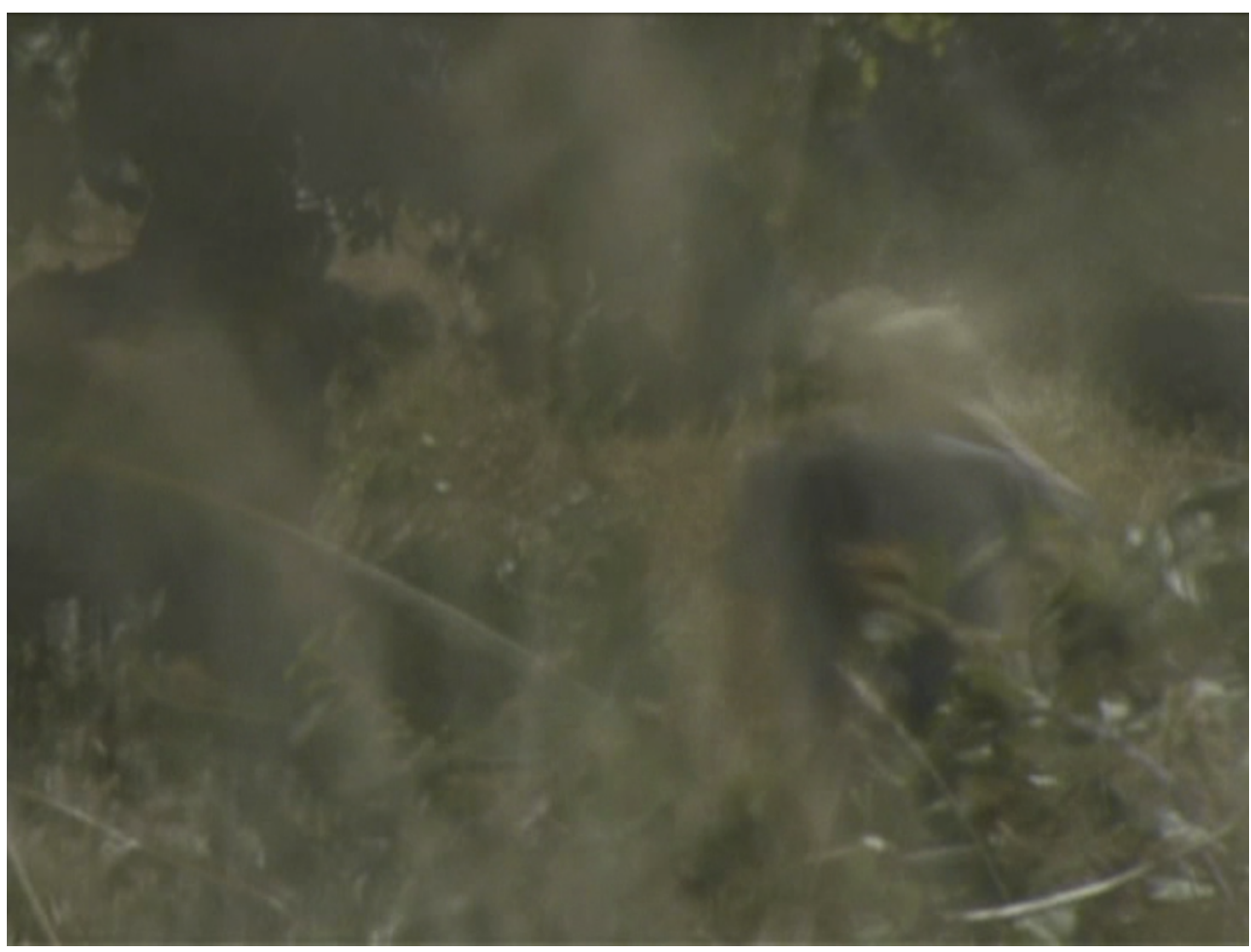

Evaporation, Leighton Pierce (2002)

6 Créant un prolongement et une figuration singulière de cette «philosophie de la fluidité, où rien ni personne ne sont ce qu'ils sont, mais deviennent ce qu'ils deviennent ${ }^{15}$ ", Evaporation répond à cette fonction de la culture de soi que relève Foucault et qui doit permettre non seulement d'acquérir de nouvelles connaissances mais aussi «[d]ésapprendre (...) qui est une des tâches importantes du développement de soi. ${ }^{16}$ ». Le souci de soi que pratique Pierce, directement lié à une relation d'amour personnelle et paternelle à ses enfants, s'exprime au travers de l'assemblage inédit d'expérimentations techniques de captation et de montage donnant naissance à des formes plastiques radicales du flou qui visent à une description plus exacte de soi par les relations filiales.

7 Sabine Massenet, plasticienne et vidéaste, pratiquant le numérique depuis la fin des années 1990, offre avec Transports amoureux (2003-2005) une variation technique sur le verre trouvé, essentielle par sa concision, sa force plastique et émotionnelle, proposant une concrétion d'expériences intimes afin de révéler une expérience collective contemporaine. L'œuvre de Massenet travaille le fragment et le collage et procède de cette conviction que « les inventions formelles les plus radicales peuvent se trouver dans des œuvres d'une simplicité déconcertante $\aleph^{17}$. Avant de concevoir le film, Massenet a tout d'abord collecté pendant deux ans les petites annonces de la rubrique du même nom dans Libération, envoyées par des gens qui pensaient avoir manqué une occasion de faire la connaissance d'un-e inconnu-e rencontré-e à bord d'un transport en commun et qu'ils espéraient retrouver. Fascinée par ces annonces qu'elle découpait et collait dans ses carnets, elle n'envisageait cependant pas encore le film. Sa rencontre fortuite avec un verre spécial dans une boutique parisienne dont les propriétaires faisaient changer la vitrine a matérialisé le film devant elle : il s'agissait d'un grand écran rectangulaire de verre sablé au travers duquel la lumière réfléchie par les passants était recomposée. 


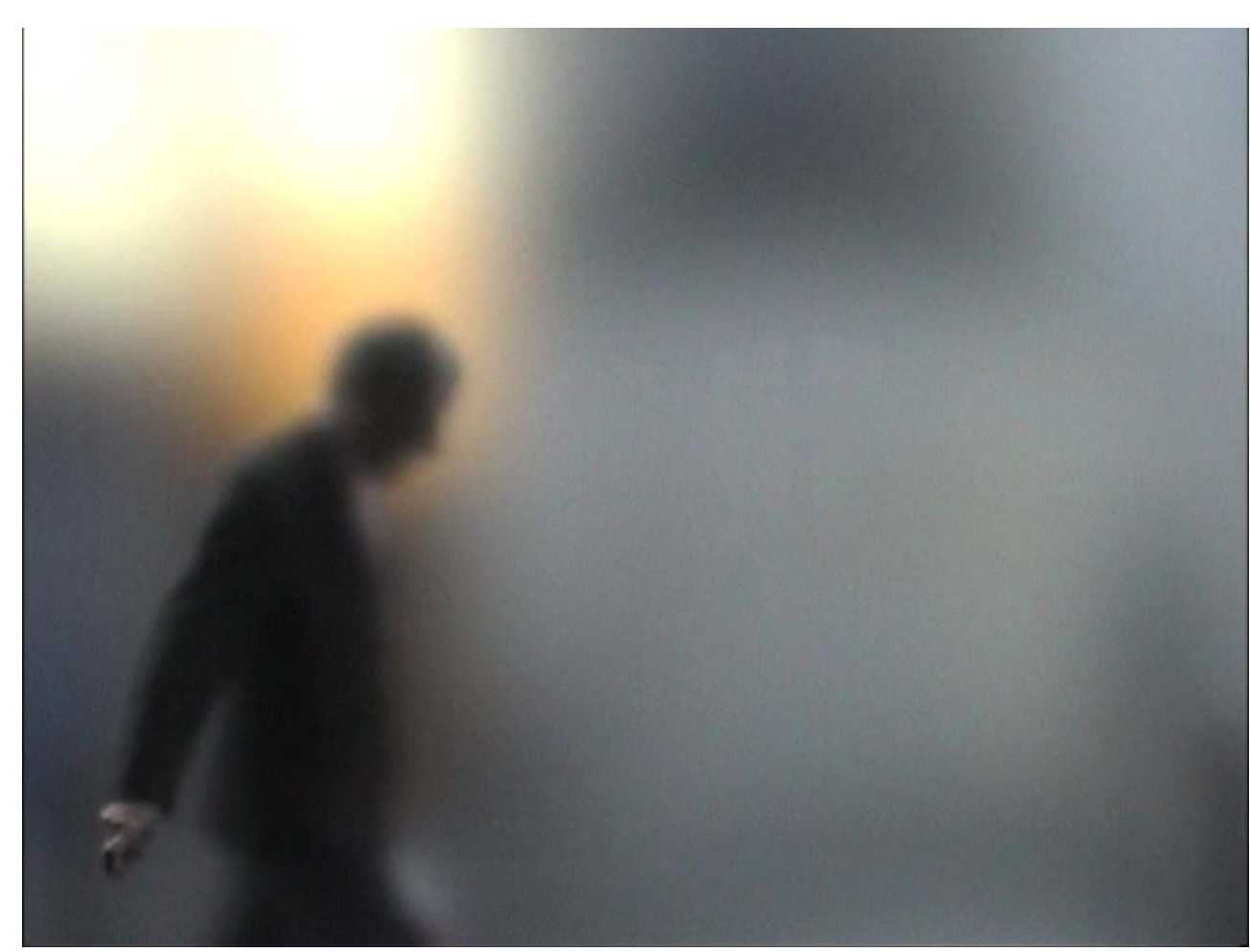

Transports amoureux, Sabine Massenet (2003-2005)

8 Massenet a placé sa mini-DV sur trépied à l'intérieur, en cadrant la vitrine, le point rigoureusement fait sur le verre et laissé tourner pendant deux heures. L'image est donc nette. Le flou vient d'une part de la profondeur de champ (au-delà du plan de mise au point, c'est de plus en plus flou) ; il est d'autre part remodelé et amplifié par les propriétés du verre agissant comme un filtre qui diffuse, diffracte et réfracte la lumière de manière forte et très complexe. Le savant montage des images arrêtées et mise en mouvement métamorphose les corps humains isolés en taches poreuses de couleurs qui se meuvent, et, par de très lents fondus, apparaissent comme l'encre dans la soie et se dissolvent en se fondant dans la toile d'une luminosité pâle. 

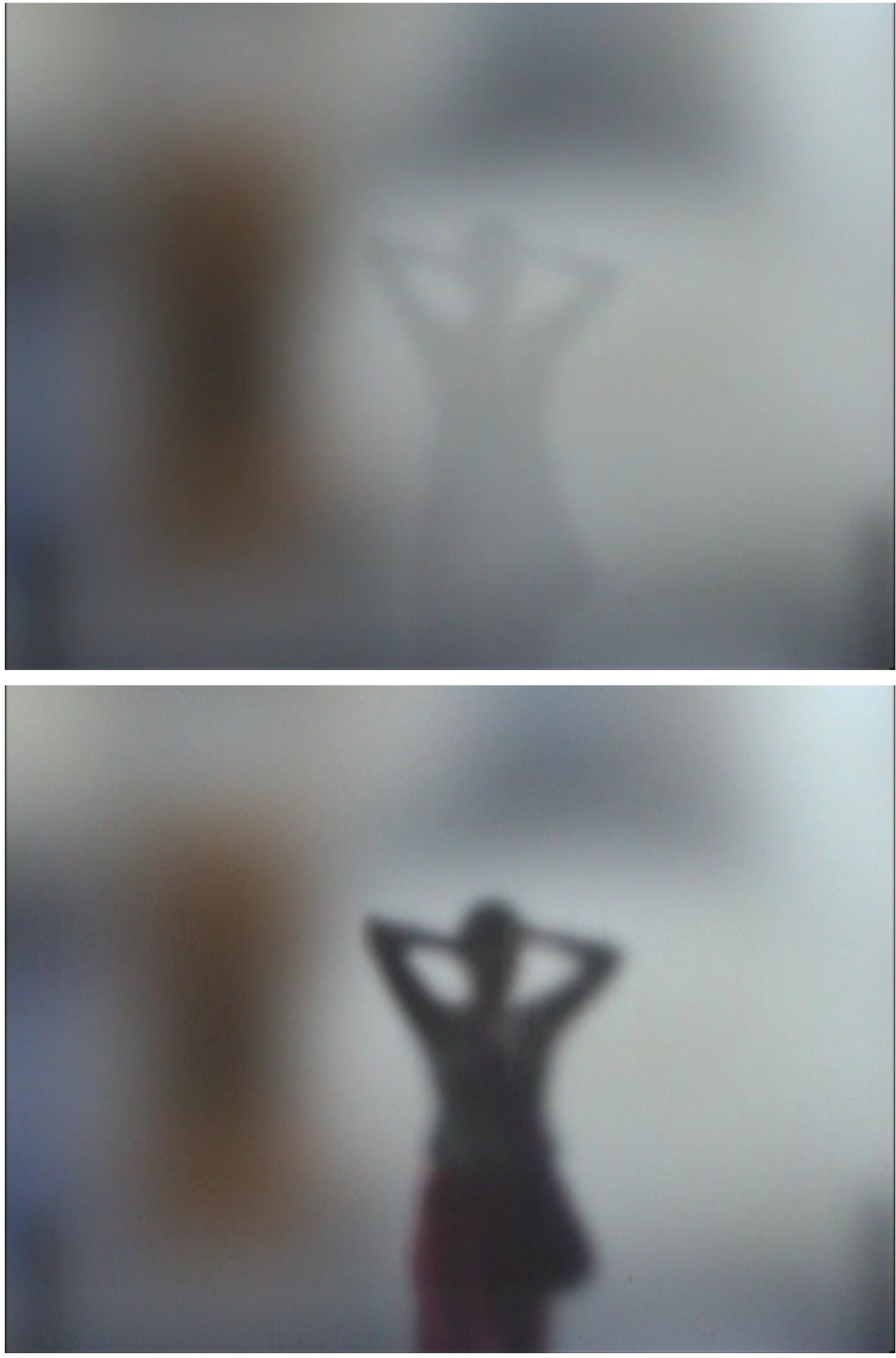

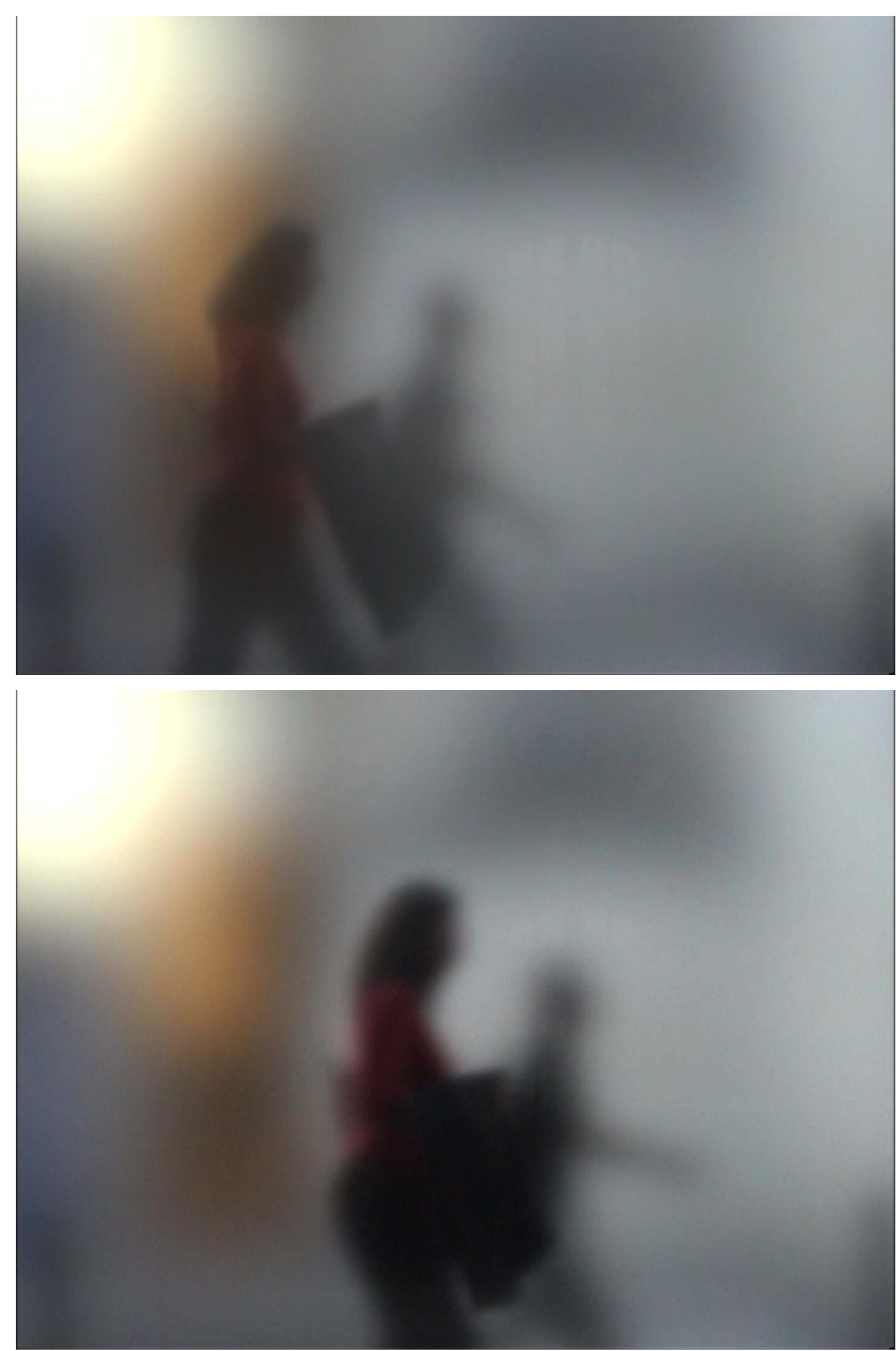

Transports amoureux, Sabine Massenet (2003-2005)

9 Massenet s'est inspirée des considérations de Marcel Proust sur la réminiscence : « Nous ne nous en souvenions déjà plus tant ce qu'on appelle se rappeler un être c'est en réalité l'oublier »; ainsi chaque entrevue (avec une personne connue, une sensation, un objet) est-elle " une espèce de redressement ${ }^{18}$ ». En optique, le redressement désigne les modifications techniques qui permettent de former une image respectant la position de l'objet, sa perspective, sa mise à l'échelle. Les tentatives de réminiscence consisteraient, 
selon Proust, moins en la réduction inéluctable du souvenir que sa transformation continue à partir d'éléments sélectionnés et/ou non avérés. Ainsi « ces rêves qui passent » en ombre colorées diffuses sont-ils soumis aux mêmes variations de quantité de flou que dans la Petite marchande d'allumettes (1928) de Jean Renoir (passage du flou au quasi net et inversement) et représentent-elles l'effort de réminiscence de la personne écrivant l'annonce par juxtaposition d'éléments fugaces non spécifiques (jour, heure, numéro de train, couleur des vêtements, des cheveux, des yeux, échange de regards, etc.) qui détermine précisément cette infinité d'images possibles de la personne évoquée. Le flou devient un liant entre le monde et les êtres ; il incarne une misère affective propre à notre société actuelle créée par ces conditions de vie paradoxale de coexistence de solitudes, d'évitement de l'autre, mais plutôt que de souligner le désespoir anonyme, il offre à ces chuchotements de sentiments naissants, aussi éphémères soient-ils, les plus belles et effusives formes plastiques qui bouleversent et transportent le spectateur.

Les techniques développées par Massenet s'élèvent du champ de ruines des techniques de soi au temps du numérique, dont les annonces collectées constituent à la fois un symptôme et un vestige. Elles organisent et composent avec bienveillance et empathie cet ensemble d'expériences intimes d'un souci de soi atrophié, pour décrire avec précision une désolation du cœur contemporaine et la transfigurer en une célébration d'un désir d'aimer et de se lier aux autres. Ainsi Transports amoureux sert-il une fonction curative et thérapeutique ${ }^{19}$.

11 Enfin, décelant la porosité et l'interconnexion nécessaire du soi à l'autre et au collectif, Jacques Perconte juxtapose très précisément les techniques qu'il invente avec un projet d'émancipation collective. Perconte, plasticien du numérique depuis 1996, ne cesse de critiquer les dernières hautes technologies par une reprise en main des outils numériques, informée par une recherche précise de leur fonctionnement et par les pratiques du cinéma expérimental et des arts plastiques qui l'ont amené à développer une stratégie de non-coopération technique.

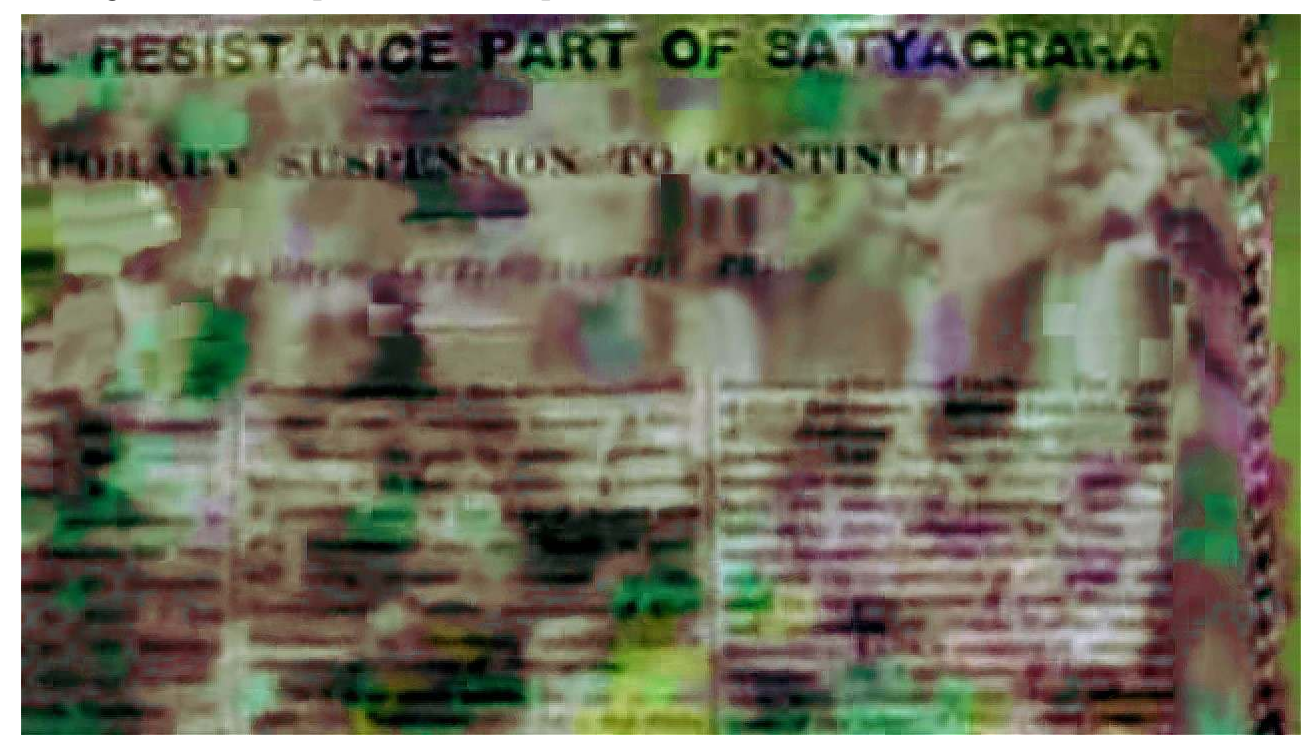

Satyagraha, Jacques Perconte (2009)

Satyagraha (2009) est sans aucun doute le film de Perconte qui explicite et porte à sa plus haute intensité cette stratégie. Il fait partie du film collectif Outrage \& Rebellion (2009, n\&b, coul., 180'), suite à une manifestation pacifiste le 9 juillet 2009 à Montreuil-sous-Bois 
s'inscrivant dans le vaste mouvement de protestation contre les arrestations de sanspapiers, leur expulsion de logement et la précarité dans laquelle ils sont maintenus, qui a été réprimée par les forces de l'ordre, et au cours de laquelle le réalisateur Joachim Gatti a été éborgné par le tir d'un flashball. Quarante-cinq cinéastes, vidéastes, musiciens et plasticiens ont répondu par les moyens du cinéma à ces actes arbitraires de violence étatique brisant la déontologie du gardien de la paix, et, par l'assemblage de leurs courtsmétrages, formé la constellation d' « un film pour les déjà trop nombreuses victimes de flashball et plus largement les cibles du néo-libéralisme anachronique qui nous mutile tous d'une façon ou d'une autre au quotidien ${ }^{20}$ ». Le titre du film collectif remet en cause et retourne deux termes de la légalité issus de la société disciplinaire - l'outrage ${ }^{21}$ est l'acte de porter atteinte à la dignité du dépositaire de l'autorité publique, la rébellion ${ }^{22}$, l'opposition d'une résistance à l'agent dans l'exercice de ses fonctions - afin d'incriminer une politique répressive. L'impérieuse nécessité d'agir contre cette politique a induit la réalisation dans l'urgence des films sans aucune production.

Les événements de Montreuil et de la Seine Saint-Denis depuis les émeutes de 2005 ont inspiré à Perconte un parallèle avec la situation de l'Inde colonisée par les Britanniques et réduite à des conditions iniques de vie rendant absolument nécessaire ce que Deleuze nomme « un devenir révolutionnaire ». Mohandas Karamchand Gandhi a façonné une puissante philosophie de résistance fondée sur la non-coopération non violente massive des Indiens, qui supposait de s'exposer délibérément aux sanctions, à l'emprisonnement et à la mort, mais selon un processus long et patient, par étapes de désobéissances successives de plus en plus émancipatrices qui a abouti à la force irrépressible du "Quit India » en 1942. Le satyagraha, littéralement " étreinte de la vérité » en sanskrit, qui peut également être interprété comme " persuasion par la seule force de la vérité ", impliquait de servir sans concession pour une cause juste afin de sevrer le système britannique de son erreur. Ainsi Perconte a-t-il pu exprimer cette analogie de la manière suivante : « J'ai trouvé dans la figure de Gandhi de nombreux liens avec les événements de Montreuil. La manifestation, la violence, la répression, l'injustice, l'engagement résistant et l'engagement du corps ${ }^{23}$ » .

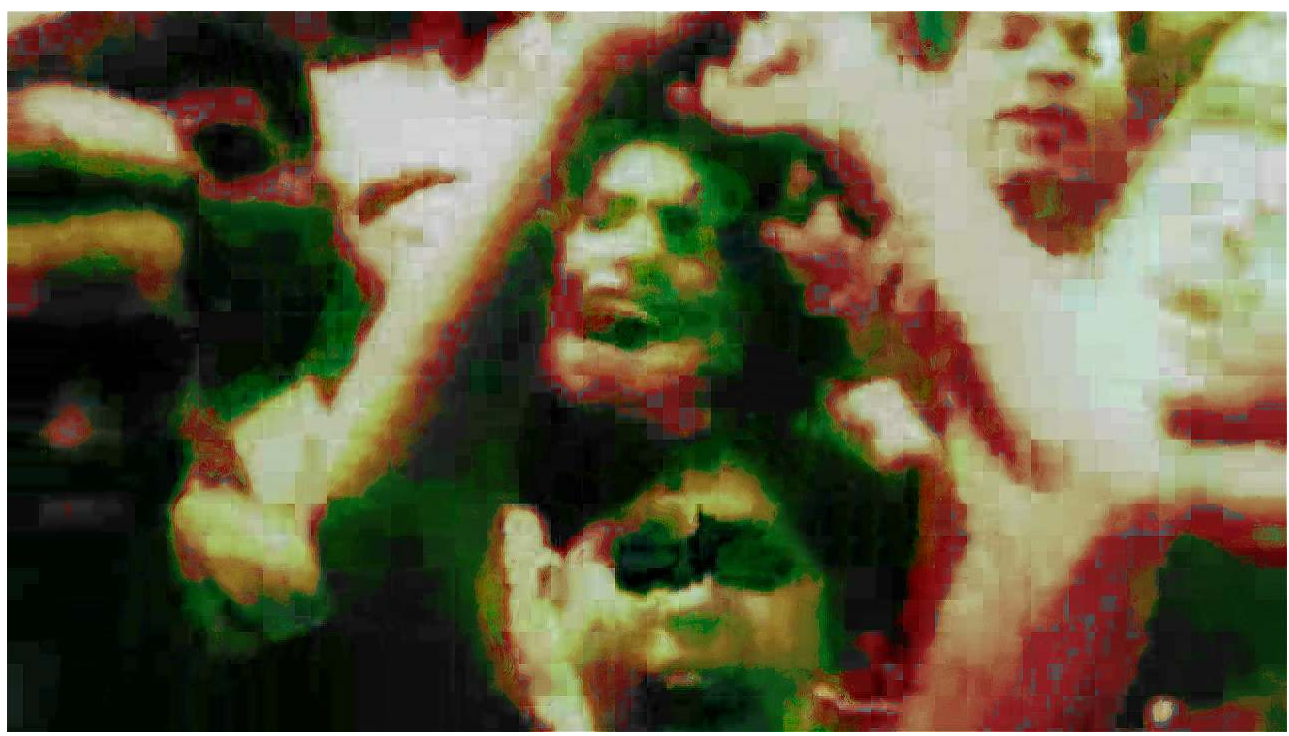

Satyagraha, Jacques Perconte (2009)

Perconte a téléchargé, depuis les banques de données des archives de la Gandhi Serve Foundation, des plans tourn és en $16 \mathrm{~mm}$ noir et blanc d'immenses manifestations 
indiennes réprimées violemment par l'armée britannique. Mais la conversion numérique des pellicules argentiques et leur compression pour le réseau a modifié la nature et la structure même des images originales : ainsi le noir et blanc argentique avait-il été codé en couleurs RVB, puisque, Gabrielle Reiner nous le rappelle, le noir et blanc n'existe pas en numérique, il ne peut être qu'imité24 ; d'autre part, cette structure de données compressées se prêtait à une reconfiguration par logiciel d'encodage. La chaîne de très fortes compressions temporelles auxquelles Perconte a soumis les signaux a révélé des couleurs sous-jacentes (teintes vertes, jaunes, rouges), et les paramétrages qu'il a opéré ont permis de restructurer les foules selon des réseaux mobiles de blocs carrés de pixels, de briser les limites des plans de manière à ce que ceux-ci s'interpénètrent, d'obtenir des traînes persistantes sur les mouvements des bras, drapeaux, corps courant. Ce faisant, les formes obtenues figurent la violence et les blessures auxquelles s'exposent les manifestants, mais aussi la force de cette multitude de corps d'Indiens de toutes conditions qui semblent n'en faire qu'un seul immense qui se répand, fulgure, ondoie, déferle, se vaporise et emporte et noie tout sur son passage. Perconte donne une forme concrète à la philosophie du satyagraha qui consiste à devoir souffrir plutôt que d'infliger la violence envers l'oppresseur, jusqu'à ce qu'il cède : c'est l'arme d'une foule forte de son nombre et de son exigence qui met un terme à l'injustice.

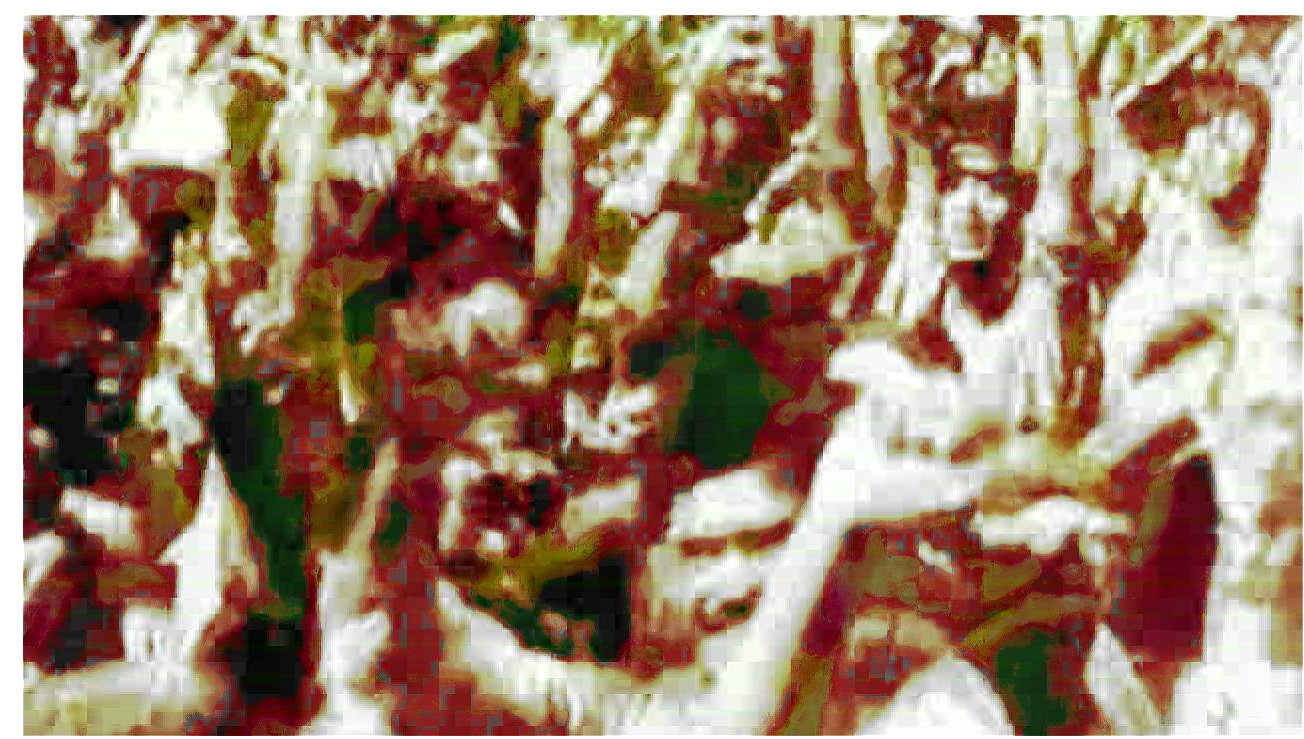



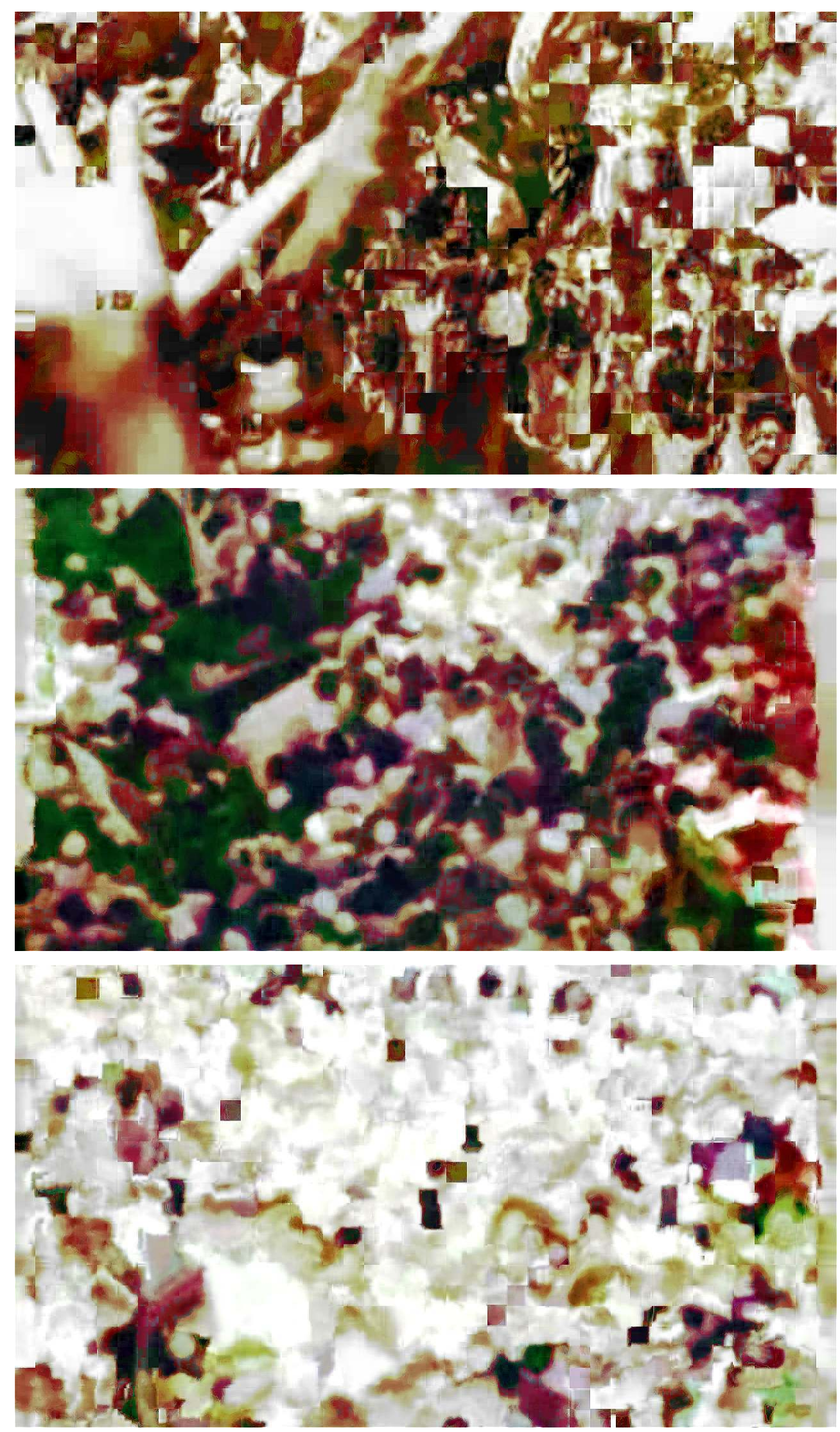

Satyagraha, Jacques Perconte (2009) 

civile envers des lois injustes - et le devoir de coopération avec le bien - s'abstenir de toute violence et propager l'amour - trouvent un équivalent dans le travail de Perconte qui correspond à un processus de non-coopération radicale technique avec la colonisation numérique et de rupture avec son idéologie : désobéissance envers les usages d'internet, des logiciels d'encodage et de l'intégrité du signal codifié qui viennent d'un long et patient développement de détournements, de refus, de radicalité, au nom d'une valeur très haute d'exigence plastique et éthique. Perconte fait preuve, grâce à cette noncoopération, d'une puissance créatrice féconde et fertile élevée au rang d'une politique formelle au service du bien commun parce qu'elle pousse à l'engagement. C'est, en effet, ainsi que nous pouvons interpréter la fin du film lorsque nous entendons une veille femme raconter un rêve dans lequel Gandhi apparaît et octroie des richesses à tous, qu'elle se réveille et se demande où est Bapu. Perconte monte sur ces paroles une citation du Mahatma : «Sois le changement que tu veux voir dans le monde » qui renvoie chacun à sa capacité à changer le monde dans lequel il vit, par la résilience et en coopérant dans le partage de valeurs communes de bonté. Les techniques développées au travers de Satyagraha répondent à une fonction de lutte selon laquelle « la pratique de soi est conçue comme un combat permanent (...) [et qu']il faut donner à l'individu les armes et le courage qui lui permettront de combattre toute sa vie. ${ }^{25} »$.

L'analyse succincte des techniques et des formes de trois films nous indique que les technologies numériques peuvent être - à condition de les soumettre à des recherches critiques - intégralement mises au service des techniques de soi. En effet, le souci de soi développé par Leighton Pierce, Sabine Massenet et Jacques Perconte décline les trois fonctions principales énoncées par Michel Foucault: désapprendre, guérir, lutter. Il s'exprime cependant au travers d'objections techniques en prises de vue et postproduction déterminant des formes plastiques radicales : les dernières techniques de soi proposées sont intrinsèquement filmiques. Aussi remarquons-nous que les inventions techniques et plastiques sont strictement analogues aux transformations récentes des techniques de soi, qu'il resterait à étudier plus en profondeur. sites privilégiés depuis lequel l'appropriation des technologies numériques correspondrait, entre autre, à une repossession de soi, et renouerait de manière très vive avec l'attitude critique selon Foucault : « L'art de n'être pas tellement gouverné ${ }^{26}{ }^{\text {». }}$

\section{BIBLIOGRAPHIE}

Nicole Brenez, Bidhan Jacobs (dir.), Le cinéma critique. De l'argentique au numérique, voies et formes de l'objection visuelle, Paris, Presses de la Sorbonne, 2010. 
Dominique Cardon (dir.), Politique des algorithmes. Les métriques du web, Paris, Editions la Découverte, Réseaux, vol 31-177, février-avril 2013.

Dominique Cardon, À quoi rêvent les algorithmes? Nos vies à l'heure des Big Data, Paris, Seuil, 2015.

Antonio A. Casilli (dir.), Cultures du numérique, Communications, $n^{\circ} 88$, Ecole des Hautes Etudes en Sciences Sociales Centre Edgar Morin, Paris, Seuil, 2011.

Grégoire Chamayou, Théorie du drone, Paris, La Fabrique éditions, 2013.

Jean Epstein, Ecrits sur le cinéma, tome 1 et 2, Paris, Seghers, 1974.

Viviane Forrester, L'Horreur économique, Paris, Librairie Arthème Fayard, 1996.

Michel Foucault, Qu'est-ce que la critique ? suivi de La culture de soi, Paris, Vrin, 2015.

Bidhan Jacobs, Vers une esthétique du signal. Dynamiques du flou et libération du code dans les arts filmiques (1990-2010), Thèse sous la direction de Nicole Brenez, Paris 3, 2014, à paraître en 2018 aux Presses du Septentrion.

François Jarrige, Technocritiques. Du refus des machines à la contestation des technosciences, Paris, La Découverte, 2016.

Catherine Malabou, Métamorphoses de l'intelligence. Que faire de leur cerveau bleu ?, Paris, PUF/ Humensis, 2017.

Evgeny Morozov, The Net Delusion. The Dark Side of Internet Freedom, New-York, PublicAffairs, 2011.

Evgeny Morozov, Le mirage numérique. Pour une politique du Big Data, Paris, Les Prairies Ordinaires, 2015.

Marcel Proust, A la recherche du temps perdu, Tome II, A l'ombre des jeunes filles en fleurs, Paris, NRF Gallimard, 1919.

Bernard Stiegler, Dans la disruption. Comment ne pas devenir fou ?, Paris, Les Liens qui Libèrent, 2016.

Michel Foucault, «The Political Technology of Individuals » (« La technologie politique des individus », Université du Vermont, octobre 1982, trad. P.-E. Dauzat), in Huck Gutman, Patrick H. Hutton et Luther H. Martin (ed.), Technologies of the Self: A Seminar with Michel Foucault, Amherst, University of Massachusetts Press, 1988, p. 145-162.

Marc Lenglet, « Le trading haute fréquence : une innovation dangereuse ? », in Comprendre les marchés financiers, Cahiers français, $\mathrm{n}^{\circ} 361$, mars-avril 2011.

Marc Lenglet, « Conflicting Codes and Coding. How Algorithmic Trading Is Reshaping Financial Regulation », in Theory, Culture \& Society, SAGE, Los Angeles, Londres, New Delhi et Singapour, Vol. 28(6), décembre 2011.

Pascal Martin, « Le flou comme paradigme du net », in Pascal Martin, François Soulages (dir.), Les frontières du flou, Paris, L'Harmattan, 2013, p. 15-40.

Jacques Perconte, entretien du dossier « Flashball et rafales d'images », propos recueillis par Ludovic Lamant et retranscrits par Pierre Lascar, Mediapart, 19 novembre 2009.

Antoinette Rouvroy et Thomas Berns, «Le nouveau pouvoir statistique. Ou quand le contrôle s'exerce sur un réel normé, docile et sans événement car constitué de corps "numériques » », in Multitudes, nº 40, janvier 2010, p. 88-103. 


\section{NOTES}

1. Bernard Stiegler, Dans la disruption. Comment de pas devenir fou ?, Paris, Les Liens qui Libèrent, 2016, p.21

2. Viviane Forrester, L'Horreur économique, Paris, Librairie Arthème Fayard, 1996, p. 158

3. Michel Foucault, «The Political Technology of Individuals » ( La technologie politique des individus "; université du Vermont, octobre 1982 ; trad. P.-E. Dauzat), in Huck Gutman, Patrick H. Hutton et Luther H. Martin (ed.), Technologies of the Self: A Seminar with Michel Foucault, Amherst, University of Massachusetts Press, 1988, p. 145-162

4. Grégoire Chamayou, Théorie du drone, Paris, La Fabrique éditions, 2013, p.287

5. Nous renvoyons en particulier aux travaux des années 2010 de Thomas Berns, Dominique Cardon, Antonio Casilli, Grégoire Chamayou, François Jarrige, Marc Langlet, Catherine Malabou, Evgeny Morozov, Antoinette Rouvroy, Bernard Stiegler.

6. Michel Foucault, «La culture de soi » (1983), dans Michel Foucault, Qu'est-ce que la critique? suivi de La culture de soi, Paris, Vrin, 2015, p. 81-98

7. Michel Foucault, « La culture de soi », ibid., p.88

8. Ibid., p.98

9. Ibid.

10. Ibid.

11. Jean Epstein analyse par exemple dans le Cinéma du diable (1947), dans Ecrits sur le cinéma, Tome 1, Paris, Seghers, 1974, p.347, que le pouvoir du cinéma étant « de faire apparaitre partout le mouvement », « [c]ette mobilisation générale crée un univers où la forme dominante n'est plus le solide qui régit principalement l'expérience quotidienne». Ainsi, «le monde de l'écran (...) constitue le domaine par excellence du malléable, du visqueux, du liquide ». Il précise plus loin, dans la partie «A seconde réalité, réalité seconde", p.404, que «[1]e solide se trouve tout à coup menacé dans sa suprématie ; il ne représente plus qu'un genre particulier d'apparences propres aux systèmes d'ordinaire expérience et d'échelle humaine, qui sont à mouvement constant ou faiblement et uniformément varié. La fluidité, réalité de l'expérience cinématographique, est aussi la réalité de la conception scientifique, qui voit, en toute substance, une structure gazeuse ».

12. Le dispositif de mesure et de quantification du flou que Pascal Martin a inventé en 2012, « le flounetoscope ", démontre que le flou n'évolue pas de manière linéaire. Il met en évidence des courbes d'évolution du flou avant et du flou arrière (qui sont des hyperboles). Ainsi, tous les objectifs se comportent de la même manière selon une courbe dissymétrique répartie autour du plan de mise au point avec, à une distance proche de 0 , une asymptote verticale tendant vers l'infini et, dans la profondeur de l'axe optique, une asymptote horizontale tendant vers une constante. Se reporter à Pascal Martin, «Le flou comme paradigme du net ", in Pascal Martin, François Soulages (dir.), Les frontières du flou, Paris, L'Harmattan, 2013, p.22. Pour une analyse détaillée de films de Leighton Pierce expérimentant les flous avant, se reporter à Bidhan Jacobs, Vers une esthétique du signal. Dynamiques du flou et libération du code dans les arts filmiques (1990-2010), Thèse sous la direction de Nicole Brenez, Paris 3, 2014, p.146-151.

13. Pour aller plus loin dans l'analyse du smearing chez Leighton Pierce, se reporter à Bidhan Jacobs, Vers une esthétique du signal, ibid., p.187-190.

14. Par exemple, Jean Epstein remarque, dans « l'Intelligence d'une machine » (1946), dans Ecrits sur le cinéma, Tome 1, op.cit., p.285, en se fondant sur les avancées des sciences physiques qui ont repensé les quatre dimensions comme des covariants inséparables, que « [l]a marche irrévocable $\mathrm{du}$ temps impose (...) à tous les mouvements de l'univers, un sens unique, une valeur irrécupérable et indestructible, perpétuellement positive. » Ainsi peut-il en déduire que «[1]a 
qualité sui generis de la dimension temporelle est un pouvoir d'orienter l'espace géométrique, de sorte que les successions ne peuvent s'y produire que selon le sens de cette polarisation. ». Les conséquences formelles et plastiques de cette découverte sont explorées dans ses films notamment grâce au ralenti et à l'accéléré ainsi qu'il l'observe page 257 : «Les chevaux planent au-dessus de l'obstacle; les plantes gesticulent; les cristaux s'accouplent, se reproduisent, cicatrisent leurs plaies ; la lave rampe ; l'eau devient huile, gomme, poix arborescente ; l'homme acquiert la densité d'un nuage, la consistance d'une vapeur ; il est un pur animal gazeux ».

15. Jean Epstein, « le Cinéma du diable », op. cit., p.406.

16. Michel Foucault, « La culture de soi », op. cit., p.93

17. Sabine Massenet, «Entretien questions communes », Annexes, dans Bidhan Jacobs, Vers une esthétique du signal. ibid., p.341

18. Marcel Proust, A la recherche du temps perdu, Tome II, A l'ombre des jeunes filles en fleurs, Paris, NRF Gallimard, 1919, p. 183

19. Michel Foucault, « La culture de soi », op. cit., p.94

20. Appel initial rédigé par Nicole Brenez et reproduit dans le programme pour la projection d'un choix de films à l'Ecole des Beaux-Arts de Paris le 3 février 2010.

21. « Les paroles, gestes ou menaces, les écrits ou images de toute nature non rendus publics ou l'envoi d'objets quelconques adressées à une personne investie d'une mission de service public dans l'exercice ou à l'occasion de sa mission et de nature à porter atteinte à sa dignité ou au respect de la fonction dont elle est investie » (article 433-5 du Code Pénal).

22. «Le fait d'opposer une résistance violente à une personne dépositaire de l'autorité publique ou chargée d'une mission de service public agissant dans l'exercice de ses fonctions, pour l'exécution des lois, des ordres de l'autorité publique, des décisions ou mandats de justice » (article 433-6 du Code Pénal).

23. Jacques Perconte, entretien du dossier « Flashball et rafales d'images », propos recueillis par Ludovic Lamant et retranscrits par Pierre Lascar, Mediapart, 19 novembre 2009.

24. Gabrielle Reiner, «Persistance du noir et blanc », dans Nicole Brenez, Bidhan Jacobs (dir.), Le Cinéma critique. De l'argentique au numérique, voies et formes de l'objection visuelle, Paris, Presses de la Sorbonne, 2010, p.244

25. Michel Foucault, « La culture de soi », op. cit., p.94

26. Michel Foucault, «Qu'est-ce que la critique?» (1978) dans Michel Foucault, Qu'est-ce que la critique? suivi de La culture de soi, op. cit., p.37

\section{RÉSUMÉS}

Les recherches technocritiques en sciences humaines ont inscrit et analysé les technologies numériques, en particulier dans les années 2010, dans l'arsenal des dernières «technologies politiques ", réinterprétant le concept développé par Michel Foucault dans les années 1970. L'hypothèse avancée dans ce travail consiste en ce que les technologies numériques auraient également capté et détourné «les techniques de soi » que Foucault entendait comme art de se conduire. Cependant, ce souci de soi se manifeste dans les œuvres de certains plasticiens d'aujourd'hui, tels que Sabine Massenet, Jacques Perconte, Leighton Pierce. Les technologies numériques semblent, chez ces artistes, constituer les fondations et les conditions d'une 
«matrice pratique de l'expérience de soi »: se faire soi-même, se transformer, revenir à soi, apprendre à vivre, prendre possession de soi, s'émanciper.

The technocritical researches in social sciences have analyzed the digital technologies, in particular during the 2010 's, as one of the "political technologies ", reinterpreting the concept developped by Michel Foucault in the 1970's. This work proposes the hypothesis that the digital technologies have also captured et deflected the "technologies of the self " that Foucault conceptualized as the art of selfconducting. However, the care of the self exists in some of the works by today's artists, such as Sabine Massenet, Jacques Perconte or Leighton Pierce. The digital technologies seams to become between their hands the foundations et the conditions of a "pratical matrix of self-experience » : to build, transform, come back to oneself, to learn to live, to take possession of and free oneself.

\section{INDEX}

Mots-clés : souci de soi, Jacques Perconte, Leighton Pierce, Sabine Massenet, technologies numériques

\section{AUTEUR}

\section{BIDHAN JACOBS}

A soutenu une Thèse à Paris 3 sous la direction de Nicole Brenez à paraître en fin 2018 aux Presses Universitaires du Septentrion sous le titre Esthétique du signal. Il a mené un post-doctorat du Labex Arts-H2H à L'Ecole Louis-Lumière de 2016 à 2017 ; il est aujourd'hui chercheur associé du Laboratoire International de Recherches en Art (LIRA) à Paris 3. Spécialisé dans les rapports entre technologie, idéologie et esthétique, il est codirecteur avec Nicole Brenez de l'ouvrage collectif Le cinéma critique. De l'argentique au numérique, voies et formes de l'objection visuelle (Publications de la Sorbonne, 2010) et collaborateur à Camera, La Furia Umana, Débordements, Turbulences vidéo. Il est actuellement enseignant à Paris 3, après avoir été ATER à l'Université Lyon 2, chargé de cours à Paris Est et intervenant dans les écoles de cinéma Georges Méliès, CLCF et l'Ecole de la Cité. 